\title{
VALIDATING THE FACTS2 AIR TRAFFIC SIMULATION FRAMEWORK
}

\author{
T. Gräupl, German Aerospace Center, Oberpfaffenhofen, Germany
}

\begin{abstract}
The modernization of the European air transportation system will increase the need for data communication. This creates the need for largescale simulations to ascertain that new aeronautical communication systems fulfil the requirements of future air traffic management communication. In this paper we validate the method of synthetic air traffic generation for aeronautical communication system evaluation by comparing the results of the FACTS2 simulator to radar-correlated flight-plan data of Germany and EUROCONTROL forecast data for Europe. FACTS2 is the German Aerospace Center's next generation framework for aeronautical communication system evaluation. Our results show that FACTS2 captures European air traffic very well, but overestimates the number of flights in Germany. The geographic distribution of flights is accurate at a geographic resolution of $0.1^{\circ}$ or coarser and the time distribution of flights in Germany is reproduced correctly.
\end{abstract}

\section{Introduction}

At the heart of the modern air traffic management system there is the communication infrastructure that enables efficient aircraft guidance and safe separation in all phases of flight. However, although current systems are mature and generally providing a good service, they are suffering from the increasing saturation in high density areas [1] [2]. The EU and US, representing the two areas experiencing the most pressure, strive therefore for the sustainable modernization of the aeronautical communication infrastructure. Air traffic management (ATM) communication shall transition from analog VHF voice communication to more spectrum efficient digital data communication supported by automated decisions of computer systems [3].

The digitization of the air-ground communications infrastructure has to be evaluated carefully against the expected future air traffic to ascertain its sustainability and future-proofness. The accepted approach to this endeavor is to employ large-scale computer simulations.
Various methods have been devised for this purpose. Khanna et al. [4] created the FASTE-CNS traffic analysis and capacity planning tool for communications. In this tool selected regions of the USA are assigned constant aircraft populations. Each aircraft is simulated as a "typical" flight which is implemented by a single "typical" flight trajectory profile. Kitaori et al. [5] used constant aircraft populations to compare the ACARS and VDL Mode 2 communication performance. Gomez and Ortiz [6] recorded air traffic movements to evaluate CPDLC over VDL Mode 2. Rokitansky et al. [7] evaluated the Iris aeronautical satellite communication technology proposal of the European Space Agency (ESA) against simulated air traffic with simplified flight trajectories generated from statistics gained from EUROCONTROL flight plan databases. The same approach was used for the evaluation of VDL Mode 2 Performance in Europe [8]. The L-band Digital Aeronautical Communication System (LDACS) has been evaluated by Ayaz et al. [9] using OMNeT++ as described by Hoffmann et al. in [10]. Hoffmann used simplified flight trajectories generated from a modified commercial flight plan database [11] similar to Rokitansky.

Due to the computational complexity of largescale aeronautical communication simulations all of the above methods use simplified models for aircraft populations and flight trajectories. The most recent approaches developed by Rokitansky and Hoffmann - generating synthetic air traffic with simplified flight trajectories from extrapolated flight plan databases - are largely accepted by the community as a good compromise between constant aircraft populations (too simplistic) and recorded real-world data (not available for the future). A combination of great-circle routes and simplified altitude profiles is deemed to provide sufficient fidelity for the simulation of long-range communication systems. However, the validity of this approach has yet to be investigated in detail. Although this investigation is certainly not possible in the general case we think that it can be performed for selected areas. 
In this paper we validate synthetic air traffic generation for aeronautical communication system evaluation by comparing the results of the FACTS2 simulator to radar-correlated flight-plan data of Germany. FACTS2 is the German Aerospace Center's next generation framework for aeronautical communication system evaluation [12]. It employs a similar air traffic simulation method as devised by Rokitansky and Hoffmann but is based on modern asynchronous distributed software architecture.

\section{Method}

Our validation of the synthetic air traffic generation approach is performed in two steps. Firstly, we generate synthetic air traffic for the year 2015 using the FACTS2 simulation framework. Second, we validate the simulation result against radarcorrelated flight-plan data from the same year.

\section{Synthetic Air Traffic Generation}

The basic concept for synthetic air traffic generation is to simulate "realistic" patterns of aircraft movement between airports using "typical" flight trajectories. The utility of this approach lies in its computational feasibility in the absence of exact data on future flight plans while providing more realistic air traffic patterns than constant aircraft populations.

Synthetic air traffic generation is a two-step process: First a suitable flight plan database is extrapolated to the target year; secondly aircraft movements are simulated according to these extrapolated airport relations with simplified trajectories.

We discuss this approach based on its implementation in the FACTS2 [12] simulation framework, as we assume it to be similar to the other implementations. This assumption is substantiated at the end of this section.

\section{Software Architecture}

The software architecture of FACTS2 is based on the concept of service oriented simulation [13], which is the application of service oriented computing to computer simulation problems.

The notion of service oriented computing is based on the loose coupling of services; each service contributing its specific capabilities to create an overall solution. A benefits chain leading to the ultimate solution is being built by the information flow from service to service independently from the underlying implementation.

The application of service oriented computing to simulation problems results in a service oriented simulation architecture based on the interaction of self-contained simulation services. This results in a number of benefits: Due to the distribution of the workload each service or tool has to deal with one particular aspect of the simulation only, making the overall solution less constraint by computational limitations and thus more scalable; parallelization is often easily achievable on service level. The decomposition of the simulation problem into selfcontained services results in less complex software units split by the separation of concerns that are easier to develop and maintain. The flexible combination of existing services enables the quick formulation of new solutions reusing existing implementations.

The FACTS2 air traffic simulation is implemented as a service oriented simulation of five interacting services: Scheduled flight database analysis, asynchronous trajectory simulation, trajectory merging, trajectory cropping to the area of interest, and statistical analysis of the final simulation result. The services are invoked by the Portable Batch System (PBS). PBS is software for job scheduling on UNIX computing grids. It takes care of interdependencies between services and tries to utilize the hardware as efficiently as possible by the parallel invocation of asynchronous service instances.

\section{Database Extrapolation}

The air traffic generation starts with the extrapolation of air traffic patterns to the target time in the future. In the case of this paper air traffic patterns are derived from a database from 2007 and the target year is 2015, because air traffic recordings of this year are available for Germany, the area of interest of our investigation.

We therefore start with the analysis of the flight plan database. FACTS2 uses the IATA ${ }^{1}$ database of worldwide scheduled passenger and cargo IFR flights for the years 2007 and 2008.

\footnotetext{
${ }^{1}$ The International Air Transport Association (IATA) is a trade association of the world's airlines.
} 
The database analysis restricts itself to two reference days: May $21^{\text {st }}-22^{\text {nd }}, 2007$. These days have been identified as typical days exhibiting an average number of daily flights. The database entries for these days were analyzed to create a statistical model of average European air traffic patterns. The flight patterns of the second day define the actual reference day for 2007. The flight patterns of the first day were included in the analysis to take over-night flights into account.

For each hour of the reference day we used the number of on-going flights between any two European airports as basis for the calculation of the hourly aircraft generation rate $\gamma_{T}(s, d)$ for each pair of source and destination airports $s$ and $d$, where $s, d \in \mathfrak{A}$, and $\mathfrak{A}$ is the set of airports in the flight plan database. $T \in\left\{T_{0}, \ldots, T_{23}\right\}$ represents the 24 hours of the reference day.

The IATA database lists only the scheduled passenger and cargo IFR flights of 2007 and 2008. In order to obtain air traffic scenarios for later years the number of IFR flights has thus to be extrapolated.

The number of IFR flights is extrapolated by applying growth factors to the air traffic generation rates $\gamma_{T}$. EUROCONTROL published per-country growth factors in [14] fig. 29 and [15] fig. 38 for four growth scenarios: Scenario A (highest growth), Scenario C, Scenario C', and Scenario D (lowest growth). These growth factors were applied to all flights departing from the same country. Growth factors for years not taken into account in the EUROCONTROL forecast were interpolated linearly from the closest values.

In order to minimize the error of the flight generation for the year 2007 and the European Statistical Reference Area (ESRA08; Figure 2) a correction factor of 0.94 has been applied to the flight generation rates.

\section{Trajectory Simulation, Merging and Cropping}

On the basis of the hourly flight generation rates $\gamma_{T}(s, d)$ and the per-country EUROCONTROL growth factors we simulated a reference day of synthetic average European air traffic for the year 2015. Air traffic over Germany was filtered from the European air traffic.
Flights were simulated in an asynchronous distributed approach. The simulation of each flight trajectory was implemented as an asynchronous retrograde time-stepped simulation where each flight is represented by a function $f(t)$ returning its state vector as a function of time

$$
f(t)=s_{t} \text { for } \alpha \leq t \leq \omega
$$

where $\mathfrak{s}_{t}$ is the state vector of the flight $f$ at time $t . \alpha$ is the start time of the flight, $\omega$ is the end time of the flight. $\mathfrak{s}_{t}$ comprises the position, altitude, and domain of the flight. The set of state vectors of all flights is denoted $\mathfrak{F}(t)$. It is generated from the union of the $\gamma_{T}(s, d)$ flights between airports $s$ and $d$.

$$
\mathfrak{F}(t)=\bigcup_{s, d \in \mathscr{U}}\left\{\begin{array}{c}
\left\{f_{i, s, d}(t) \mid t \in\left\{T_{0}, \ldots, T_{23}\right\}\right. \\
\left.1 \leq i \leq \gamma_{T}(s, d)\right\}
\end{array}\right.
$$

In the spatial domain all flights follow greatcircle routes from departure to destination airport and have simplified trapezoidal altitude profiles.

The flight domain is calculated from the current altitude ${ }^{2}$ of the flight. Flights below $\mathrm{FL}^{3} 0^{3}$ are considered to be in the airport domain (APT). Flights below FL245 are in the terminal maneuvering area (TMA), and flights above this threshold are in the en-route (ENR) domain. The only deviation from this mechanism is applied when the flight is not within any ATC sector. In this case the domain is assumed to be oceanic, remote, or polar (ORP). The current Air Traffic Control (ATC) sector of a flight is inferred from its current position and an ATC sector database obtained by the German Aerospace Center.

In the default configuration of FACTS2 it is assumed that the flight enters the simulation 30 minutes before takeoff and remains additional 15 minutes after landing in the simulation at the airport. However, for this paper FACTS2 has been configured not to simulate airport sojourn times, as they are not captured in the available radar data.

All other parameters of the flight are uniformly randomly distributed $+/-10 \%$ around a configurable average value: Climb/descent angle $\left(15^{\circ} / 3^{\circ}\right)$, speed

\footnotetext{
2 The altitude levels correspond to the altitude levels assumed in [18].

3 Flight Leve (FL) $=100 \mathrm{ft}$ altitude.
} 
during climb $(75 \mathrm{~m} / \mathrm{s})$, cruise speed $(260 \mathrm{~m} / \mathrm{s})$ speed during descent $(65 \mathrm{~m} / \mathrm{s})$, and cruise altitude (FL360).

An illustration of the flight state vector $s_{t}$ as a function of time $t$ is displayed in Figure 1 .

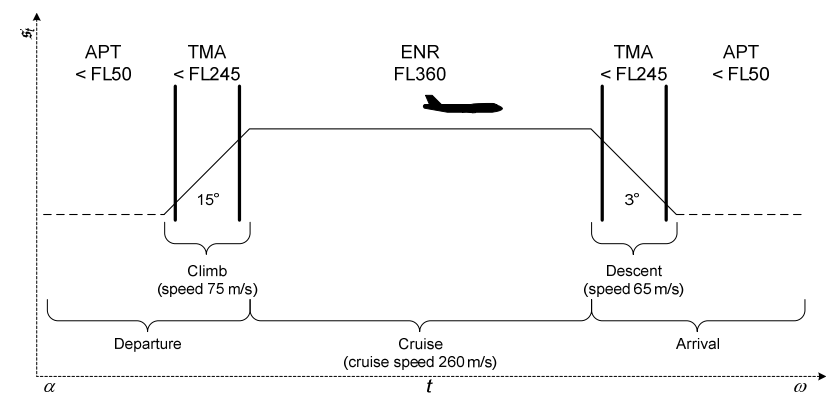

Figure 1: Illustration of the flight state vector $\mathfrak{s}_{t}$ as function of time $t$. The position component of the state vector $\mathfrak{s}_{t}$ is not displayed.

The simulation of the flight trajectories builds on the work of Hoffmann [10] [11] and Rokitansky [7] [16] [17]. All three simulators assume great circle routes with varying flight speed, pre-flight, and post-flight times at the airport. A comparison table of the simulation parameters is displayed in Table 1 providing evidence for the similarity of the approaches.

Table 1: Comparison of simulation fidelity

\begin{tabular}{rcccc}
\hline & $\begin{array}{c}\text { FACTS2 } \\
\text { (here) }\end{array}$ & $\begin{array}{c}\text { FACTS2 } \\
\text { (default) } \\
{[12]}\end{array}$ & $\begin{array}{c}\text { Hoffmann } \\
{[10]}\end{array}$ & $\begin{array}{c}\text { Rokitansky } \\
{[7]}\end{array}$ \\
\hline $\begin{array}{r}\text { Great circle } \\
\text { routes }\end{array}$ & + & + & + & + \\
$\begin{array}{r}\text { Take-off time } \\
\text { at airport }\end{array}$ & 0 & $30 \mathrm{~min}$ & - & $30 \mathrm{~min}$ \\
$\begin{array}{r}\text { Landing time } \\
\text { at airport }\end{array}$ & 0 & $15 \mathrm{~min}$ & - & $15 \mathrm{~min}$ \\
$\begin{array}{r}\text { Flight speed } \\
\text { Flight do- }\end{array}$ & $\begin{array}{c}75,260, \\
65 \mathrm{~m} / \mathrm{s}\end{array}$ & $\begin{array}{c}75,260,65 \\
\mathrm{~m} / \mathrm{s}\end{array}$ & $260 \mathrm{~m} / \mathrm{s}$ & variable \\
$\begin{array}{r}\text { main accord- } \\
\text { ing to altitude }\end{array}$ & + & + & + & + \\
\hline
\end{tabular}

\section{Validation Approach}

The synthetic air traffic generation approach is validated against radar-correlated flight-plan data of the German air navigation service provider Deutsche Flugsicherung (DFS).

Table 2 presents the number of flights per day reported by EUROCONTROL and DFS. The EUROCONTROL forecast data has been published in 2013 in [15] fig. 38. No updated data is available, yet. Note that EUROCONTROL provides the expected yearly number of IFR flights for Germany. This number has been interpolated for the year 2015 and has been divided by 365 days to yield an estimate for the daily number IFR flights in Germany forecast for 2015. The DFS data has been recorded on Jul. $30^{\text {th }} 2015$. It therefore represents the "ground truth" of our validation. Note that the EUROCONTROL forecast for Scenario A 2015 is virtually identical to the true air traffic. Our validation will therefore focus only on this scenario.

Table 2: Air traffic data for Germany 2015.

\begin{tabular}{rrrr}
\hline Flights/day & $\begin{array}{l}\text { EUROCONTROL forecast } \\
\text { for Germany 2015 [15] }\end{array}$ & \multicolumn{2}{l}{$\begin{array}{l}\text { DFS Radar-correlated } \\
\text { flight-plan data for } \\
\text { 2015 Jul. 30 }\end{array}$} \\
\hline DFS & & & 9,182 \\
Scenario A & 9,121 & & \\
Scenario C & 8,807 & \\
Scenario C' & 8,807 & \\
Scenario D & 8,382 & \\
\hline
\end{tabular}

We validate the simulated air traffic against the real air traffic in three ways: Firstly we compare the number of flights reported for each data set. Second, we compare the geographic distribution of flights in Germany. Third, we investigate the distribution of the flights in time over the duration of one day.

\section{Results}

Table 3 gives the simulation error relative to three reference sources. The ESRA08 column presents the simulation error for the ESRA08 area as displayed in Figure 2. The second and third columns present the simulation error for the number of flights reported by EUROCONTROL and DFS. The error is given for forecast Scenario A, which matches the true air traffic best.

Table 3: Error for the simulated number of daily flights relative to EUROCONTROL and DFS data for 2015.

\begin{tabular}{|c|c|c|c|}
\hline $\begin{array}{r}\text { Error for } \\
\text { number of } \\
\text { flights per day } \\
2015\end{array}$ & $\begin{array}{r}\text { Simulation vs. } \\
\text { Eurocontrol } \\
\text { forecast for } \\
\text { ESRA08 } 2015 \\
{[15]}\end{array}$ & $\begin{array}{r}\text { Simulation vs. } \\
\text { Eurocontrol } \\
\text { forecast for } \\
\text { Germany } 2015 \\
{[15]}\end{array}$ & $\begin{array}{r}\text { Simulation vs. } \\
\text { DFS Radar- } \\
\text { correlated } \\
\text { flight-plan data } \\
\text { for } 2015 \text { Jul. } \\
30^{\text {th }}\end{array}$ \\
\hline Scenario A & $0.91 \%$ & $22.32 \%$ & $21.51 \%$ \\
\hline
\end{tabular}




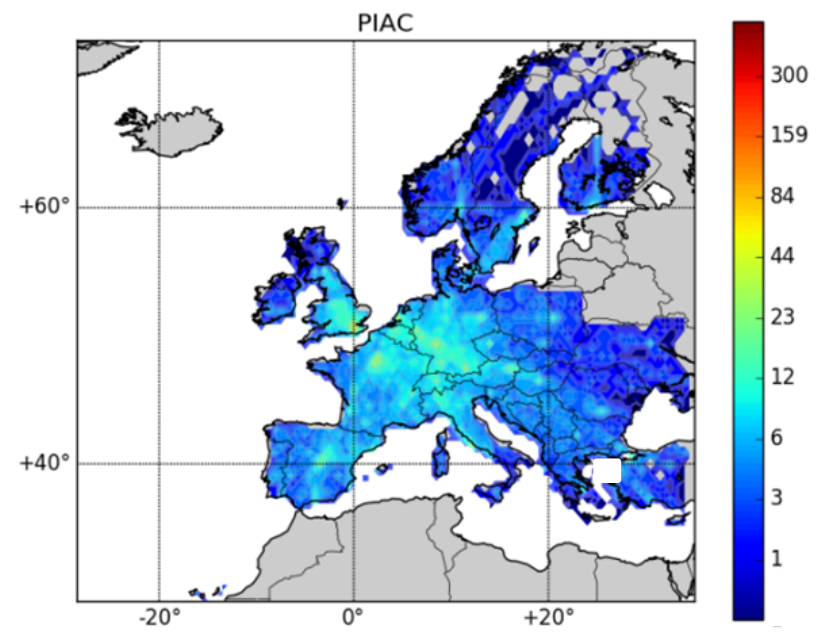

Figure 2: (left) Peak Instantaneous Aircraft Count (PIAC) $=\max _{t}|\mathfrak{F}(t)|$ of the simulation result for 2015 Scenario $A$ with a geographic resolution of $0.5^{\circ} \times \mathbf{0 . 5}^{\circ}$ degree for the European Statistical Reference Area (ESRA08).
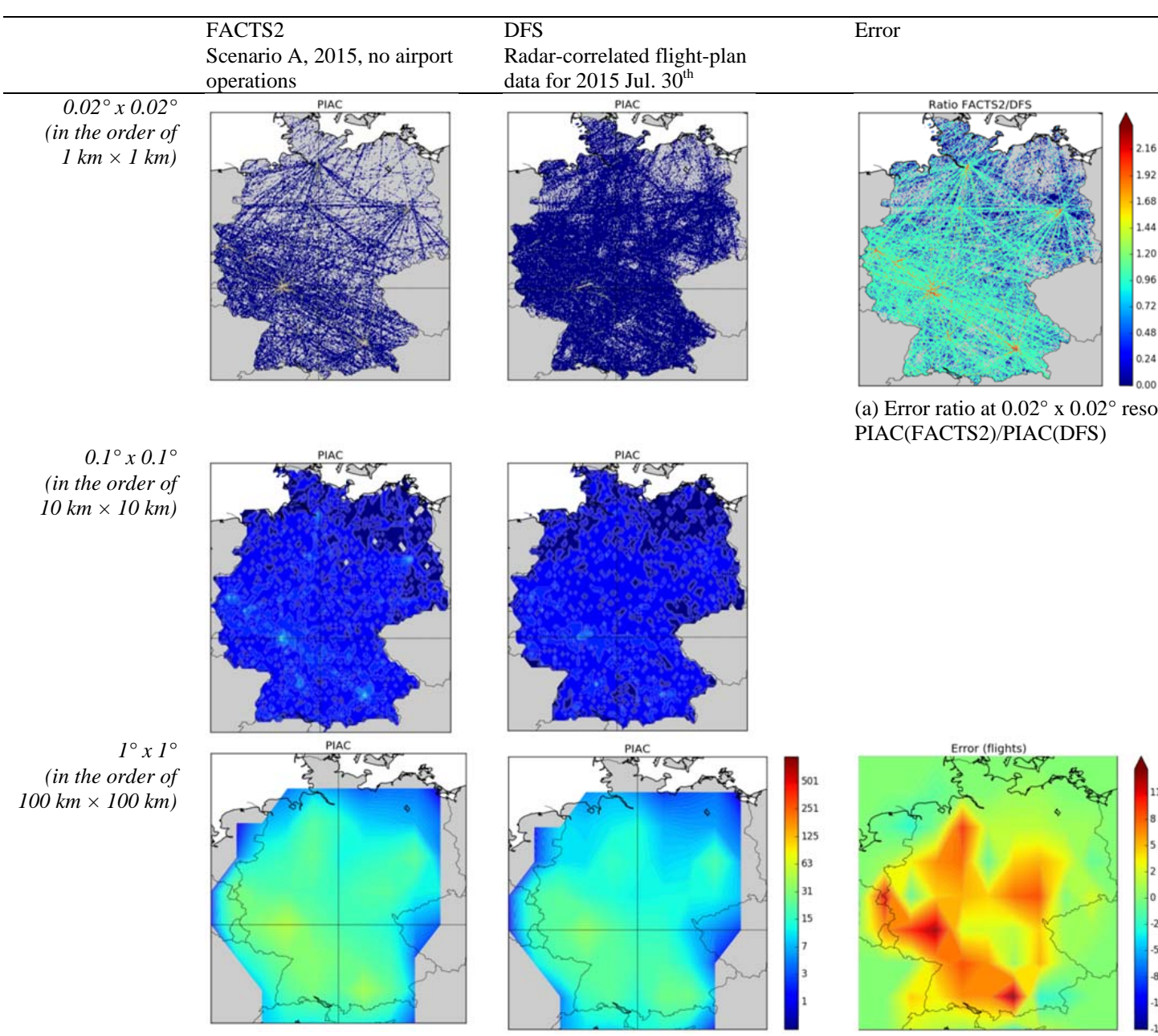

(a) Error ratio at $0.02^{\circ} \times 0.02^{\circ}$ resolution: PIAC(FACTS2)/PIAC(DFS)

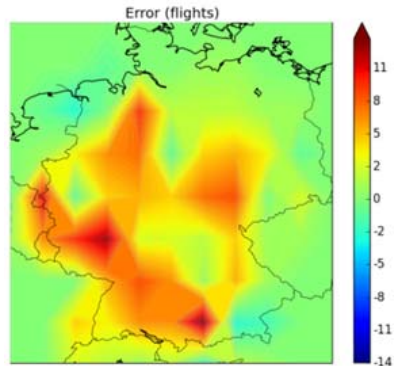

(b) Absolute error at $1^{\circ} \times 1^{\circ}$ resolution: PIAC(FACTS2) - PIAC(DFS)

Figure 3: PIAC of FACTS2 Scenario A and DFS radar-correlated flight-plan data at 0.02, 0.1, and 1.0 degrees of geographic resolution. The right column displays the (a) relative and (b) absolute error at a geographic resolution of 0.02 and 1.0 degrees, respectively. 
Figure 3 displays the geographic distribution of flights within Germany for the duration of one day. The left column shows the distribution of the synthetic air traffic generated by the computer simulation for Germany in 2015. The middle column shows the distribution of the flight traffic recorded on Jul. $30^{\text {th }}$ 2015 by DFS. Both results are given in three different geographic resolutions: $0.02 \times 0.02$ degrees, $0.1 \times 0.1$ degrees, and $1.0 \times 1.0$ degrees. For the geographic latitudes of Germany this is equivalent to a resolution in the order of kilometers, tens of kilometers, and hundreds of kilometers.

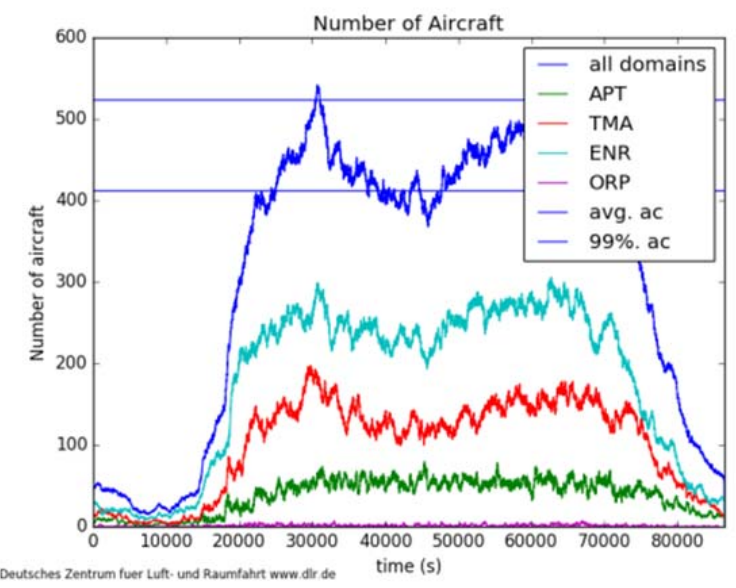

Figure 4: Number of instantaneously airborne flights over Germany for 24 hours: FACTS2 Scenario $A, 2015$, no airport operations

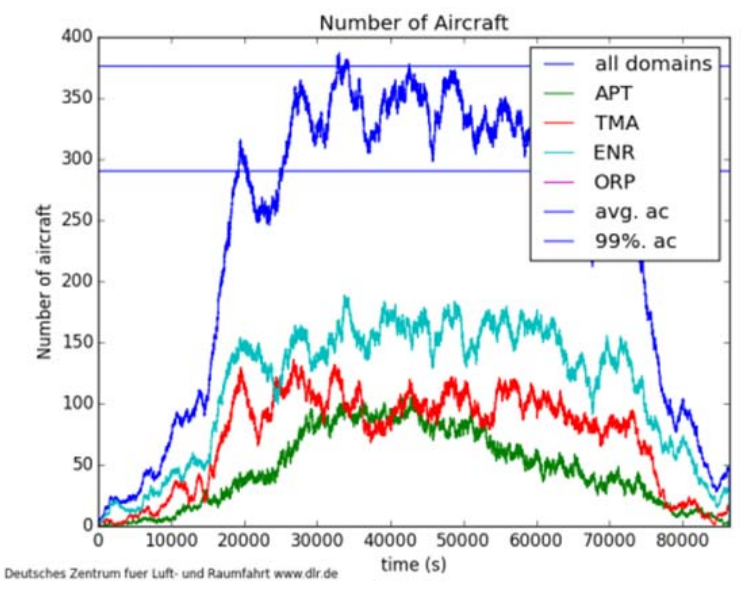

Figure 5: Number of instantaneously airborne flights over Germany for 24 hours: DFS Radarcorrelated flight-plan data for $2015 \mathrm{Jul} .30^{\text {th }}$
Figure 4 and Figure 5 display the time distribution of the flights during 24 hours. The total of all flights instantaneously airborne over Germany (all domains) is broken down into the number of airborne flights in the airport (APT), terminal-maneuvering area (TMA), and en-route (ENR) domain. The domains are only determined by the current flight altitude (APT < FL50 < TMA < FL245 < ENR) and were determined via post-processing of the DFS data.

\section{Discussion}

FACTS2 captures European air traffic very well. The error of the simulated number of flights is $0.91 \%$ relative to number of flights forecast by EUROCONTROL for 2015 Scenario A in Table 3. The error for other years and growth scenarios in reported in [12] and is generally below $3 \%$.

FACTS2 overestimates the number of flights in Germany. For Scenario A, the EUROCONTROL forecast scenario fitting the number of flights recorded by DFS best, the simulation error for the daily number of flights is $22.32 \%$ relative to EUROCONTROL data and $21.51 \%$ relative to DFS data in Table 3 i.e. FACTS2 simulates approximately 2,000 daily flights to many in Germany. The air traffic simulation is based on scheduled flight plan data for 2007 and extrapolated to the future from this year. The analysis of the error of IFR flights for the year 2007 indicates that the error for Germany is already present in the original data for Germany although it is virtually error free for Europe: The error of FACTS2 vs. EUROCONTROL data for 2007 [15] is $-2.13 \%$ for the ESRA08 area, and 20.89\% for Germany. This indicates that our air traffic extrapolation method does not introduce additional errors, but that the flights in the IATA scheduled flight database for Germany do not correspond exactly to the flights recorded by DFS in Germany. Figure 6 indicates that the additional 2,000 flights in Germany may be the largest error in the ESRA08 countries relative to the EUROCONTROL forecast Scenario A for 2015. However, it should be noted that only the forecast for Germany has been validated against true flight data in this paper. Figure 6 can therefore provide only an indication that the simulation error may actually be much lower in most cases. 


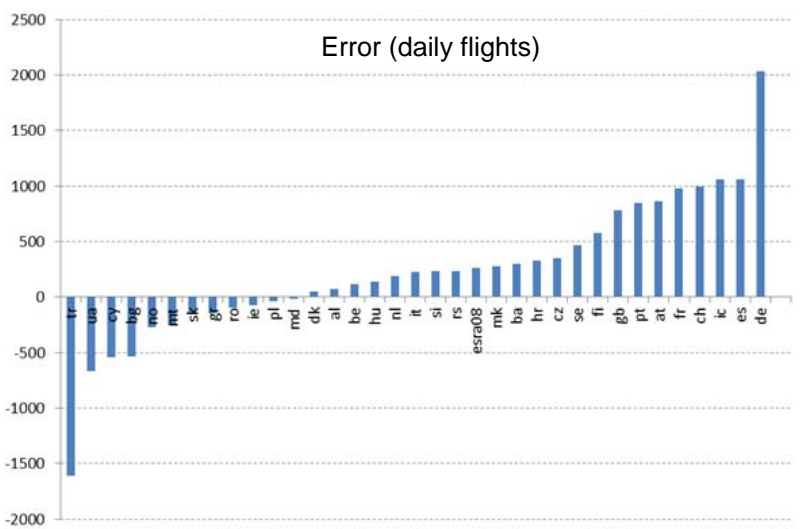

Figure 6: Error of daily flights of FACTS2 vs. EUROCONTROL forecast Scenario A for 2015 for the ESRA08 countries.

FACTS2 captures the geographic distribution of flights in Germany very well at a geographic resolution of $0.1^{\circ}$ or coarser. At a geographic resolution of $0.02^{\circ}$ Figure 3 (a) shows clearly that approximating flight trajectories by great-circle routes concentrates flights stronger than in reality. However, the absolute error of the peak instantaneous aircraft count is always less than 14 aircraft at a geographic resolution of $1.0^{\circ}$ and concentrates around the major German airports in Figure 3 (b). E.g. Frankfurt airport and Munich airport are easily discernable in Figure 3 (a) and (b). This is due to the concentration of flights through great-circle routes.

FACTS2 captures the time distribution of flights in Germany very well. Figure 4 and Figure 5 shows that the peak hours German air traffic between 6:00h and 20:00h (approximately $20.000 \mathrm{~s}$ and $70.000 \mathrm{~s}$ in Figure 4 and Figure 5) are reproduced by the simulation and that the distribution of flights to the APT, TMA, and ENR domain are captured, too. It should be noted, however, that the DFS data starts with 0 flights at the beginning of the day. This is an indication that flights of the previous day were not taken into account in the radar-correlated flight-plan data. It is an item of future work to clarify to which extent this contributes to the diverging number of flights in the simulation and the DFS data for Germany.

Can synthetic air traffic generation be used in the validation of aeronautical communication systems? In our opinion the answer is decidedly: Yes! However, as with all powerful methods some caveats have to be taken into account. Although synthetic air traffic generation is almost exact at very large scales
(ESRA08) and over very long times (2007-2015), significant deviations may emerge locally. Evaluations of aeronautical communication systems should therefore make certain to use sufficiently diverse air traffic samples from different regions. It should also be taken into account, that synthetic air traffic generation based on great-circle routes is only meaningful at geographic resolutions of $0.1^{\circ}$ or coarser. As this corresponds to a geographic resolution in the order of tens of kilometers, this method should only be used for long range or satellite-based aeronautical communication systems.

\section{Conclusion}

The objective of this paper was to validate synthetic air traffic generation for aeronautical communication system evaluation by comparing the results of the FACTS2 simulator to radar-correlated flightplan data of Germany. The results of this comparison indicate that FACTS2 is almost exact at very large scales and over very long times. However, deviations can emerge locally e.g. for Germany in this paper. If the identified limitations of the method are taken into account, synthetic air traffic generation is, however, a powerful and sound evaluation tool.

\section{References}

[1] B. Kamali, "An overview of VHF civil radio network and the resolution of spectrum depletion,” in 2010 Integrated Communications, Navigation, and Surveillance Conference Proceedings, ICNS 2010, 2010.

[2] C. Gandolfi, E. Mora-Castro, J. Myllarniemi, and F. Copigneaux, "Technical issues in the implementation of Regulation (EC) No 29/2009 (Data Link),” 2014.

[3] J. P. McGee, R. Parasuraman, A. S. Mavor, C. D. Wickens, and others, The Future of Air Traffic Control:: Human Operators and Automation. National Academies Press, 1998.

[4] M. Khanna, C. Dhas, C. Wargo, S. Vidyanandan, M. Joseph, C. Netto, and K. Wargo, "Results of field trials and features of FASTE-CNS-a traffic analysis and capacity planning tool for communications, navigation and surveillance," in Digital Avionics Systems Conference, 2003. DASC'03. The 22nd, 2003, 
vol. 1, p. 4--B.

[5] J. Kitaori, "A performance comparison between VDL mode 2 and VHF ACARS by protocol simulator," in Digital Avionics Systems Conference, 2009. DASC'09. IEEE/AIAA 28th, 2009, p. 4--B.

[6] L. Gomez and J. Ortiz, "Modeling and simulation of VDL mode 2 subnet for CPDLC in El Dorado airport," in Digital Avionics Systems Conference (DASC), 2013 IEEE/AIAA 32nd, 2013, p. 3B4--1.

[7] C.-H. C.-H. Rokitansky, M. Ehammer, and T. Gräupl, "Communication capacity assesment for the Iris satellite system," in Digital Avionics Systems Conference, 2008. DASC 2008. IEEE/AIAA 27th, 2008, p. 2--B.

[8] M. Carandente and C.-H. Rokitansky, "VDL Mode 2 Capacity and Performance Analysis," $2015 . \quad$ [Online]. Available: http://www.sesarju.eu/sites/default/files/docu ments/news/SJU_VDL_Mode_2_Capacity_an d_Performance_Analysis.pdf.

[9] S. Ayaz, F. Hoffmann, R. German, and F. Dressler, "Analysis of deficit round robin scheduling for future aeronautical data link," in IEEE International Symposium on Personal, Indoor and Mobile Radio Communications, PIMRC, 2011, pp. 18091814.

[10] F. Hoffmann, C. Bauer, D. Medina, and S. Ayaz, "FACTS: An OMNeT++ Based Simulator for Aeronautical Communications," in Proceedings of the 1st International Conference on Simulation Tools and Techniques for Communications, Networks and Systems \& Workshops, 2008, p. 85:1-85:4.

[11] F. Hoffmann, U. Epple, M. Schnell, and U. Fiebig, "Feasibility of LDACS1 cell planning in European airspace," in Digital Avionics Systems Conference (DASC), 2012 IEEE/AIAA 31st, 2012, p. 5E1-1-5E1-13.

[12] T. Gräupl, "FACTS2: A service oriented simulation framework for aeronautical communication system evaluation,” in 2016 IEEE/AIAA 35th Digital Avionics Systems
Conference (DASC), 2016, pp. 1-7.

[13] M. Ehammer, T. Gräupl, and C. H. Rokitansky, "Applying SOA concepts to the simulation of aeronautical wireless communication," in Proceedings of the 11th communications and networking simulation symposium, 2008, pp. 194-201.

[14] EUROCONTROL, "Long-Term Forecast: IFR Flight Movements 2010-2030,” 2010. [Online]. Available: http://www.eurocontrol.int/articles/challenges -growth.

[15] EUROCONTROL, "Challenges of Growth 2013 - Task 4: European Air Traffic in 2035,” $2013 . \quad$ [Online]. Available: http://www.eurocontrol.int/articles/challenges -growth.

[16] C.-H. Rokitansky, M. Ehammer, and T. Gräupl, "NEWSKY--Novel simulation concepts for future air traffic," Proc. 1st CEAS Eur. Air Sp. Conf., pp. 611-618, 2007.

[17] C.-H. Rokitansky, M. Ehammer, and T. Gräupl, "NEWSKY - Building a simulation environment for an integrated aeronautical network architecture," in AIAA/IEEE Digital Avionics Systems Conference - Proceedings, 2007, p. 4B41-4B411.

[18] EUROCONTROL and FAA, "Communication Operating Concept and Requirements for the Future Radio System, Ver. 2.0,” 2007.

\section{Acknowledgements}

The author likes to thank his colleagues from DLR, Okuary Osechas and Michael Schnell, for their support and valuable discussions.

\section{Email Addresses}

Thomas.Graeupl@dlr.de

\section{Integrated Communications Navigation and Surveillance (ICNS) Conference}

April 18-20, 2017 